MATEC Web of Conferences 22,02026 (2015)

DOI: $10.1051 /$ matecconf/ 20152202026

(C) Owned by the authors, published by EDP Sciences, 2015

\title{
Power Enhancement of Partial Shaded PV Array by Optimizing the Electrical Connection of Module
}

\author{
Mengyao Wang \\ School of Electronic and Technology, Shenzhen University, Shenzhen, Guangdong Province, China
}

\begin{abstract}
The maximum output power (Pmax) generated from photovoltaic (PV) array will be apparently reduced if the array is partially shaded. In order to enhance Pmax generated from partial shaded PV array, several interconnection schemes of array are proposed. Among these schemes, the totally cross tied (TCT) scheme and the recently proposed static scheme are widely discussed. It was reported that Pmax produced with static scheme is equal to the TCT scheme even under worst conditions. However, in these simulations the illumination of every single module is assumed to be uniform, but in urban environments the illumination of modules on the edge of shadow is more likely to be non-uniform. In this paper, first, a comprehensive circuit-level simulation, which is implemented in PSpice, has been done to investigate performance of PV array with both TCT scheme and static scheme under different partial shading conditions. And the results show that Pmax generated from static scheme is higher than that form TCT scheme if the illumination of every single module is uniform, however if some modules are partially shaded, the Pmax with static scheme is more likely less than that with TCT scheme. Then, the electrical connection of a module is improved for the purpose of enhancing Pmax under partial shading conditions in which some modules in the array are partially shaded. And the simulation results show that Pmax is apparently increased by employing the improved modules.
\end{abstract}

Keywords: photovoltaic array; maximum output power; static interconnection scheme; PSpice

\section{INTRODUCTION}

Today's grid-connected photovoltaic systems are frequently mounted on building roofs, facades, or generally in urban environments, where partial shading can be common $[1,2]$. Partial shading causes power losses through different mechanisms. For example, if one cell of the solar energy generator composed of seriesconnected cells is shaded, the shaded cell will be reversely biased. And in this case, the reversely biased cell acts as a load in the series connection dissipating part of the power generated by the other cells and results in power losses. Also, under partial shading conditions, the conventional maximum power point (MPP) tracking techniques fail to guarantee successful tracking of the global MPP, leading to significant reduction of the generated power [3-5]. However, the incoherence of array's MPP with modules' MPPs is considered to be the most severe one. This means that the MPP operation of the array does not coincide with the MPPs operation of the individual modules; therefore, the overall operation is not optimal and the maximum output power $\left(P_{\max }\right)$ of generator is reduced $[6,7]$.

It has been confirmed that $P_{\max }$ of partially shaded photovoltaic arrays could be enhanced by optimizing the interconnections among its modules [8-15]. There are four typical interconnections, "series-parallel" (SP), "bridge-link" (BL), "totally cross tied" (TCT) and "honey comb" (HC). A detailed study has been done to investigate how these typical interconnections impact power production of a partial shaded photovoltaic array [11]. And the TCT scheme is considered to be the better scheme for reducing the losses under most of the shading conditions, although none of the schemes are found to be effective under wide shading conditions. Following, the static interconnection schemes are proposed, which is improved from TCT scheme and designed to show good performance through distributing shading effect over the array. Also, it is designed without switches, sensors and complicated control algorithms to make sure it is much economic than the electrically array reconfiguration scheme $[14,15]$. In $[14,15]$, the illumination on every single module is assumed to be uniform. However, in urban environment, the illumination of modules on the edge of the shadow is more likely to be non-uniform. And this should be taken into account in the prediction of generated power from photovoltaic plant.

In this study, first, a conventional module consisting of 60 solar cells is employed to obtain the $P_{\max }$ generated from a $3 \times 3$ photovoltaic array under different partial shading conditions and with both TCT and the static interconnection schemes. And the results show that if the illumination of every single module is uniform, $P_{\max }$ generated from array with static scheme is higher than that from array with TCT scheme. However if the illumination of some modules are non-uniform, the $P_{\max }$ generated form array with static scheme is more likely less than that from array with TCT scheme. Then, the electrical connection of a 


\section{MATEC Web of Conferences}

module is improved for the purpose of enhancing $P_{\max }$ from array with static scheme and under partial shading conditions of which some modules are partially shaded. And the simulation results show that $P_{\max }$ is apparently increased by employing the improved modules.

\section{THEORY, SYSTEM DESCRIPTIONS AND METHOD}

\subsection{Equivalent circuit model of a solar cell}

Solar cells are usually represented by a one-diode circuit model [18], shown in Figure1.

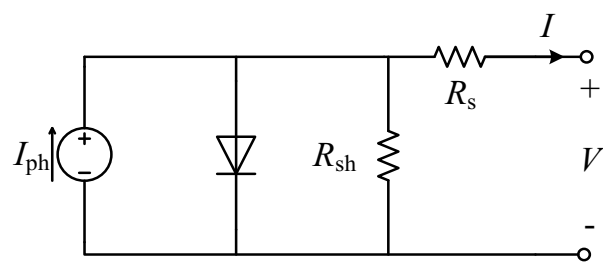

Figure 1. One-diode circuit model of a solar cell.

In the model, a shunt resistance $R_{\mathrm{sh}}$ represents leakage and recombination's current paths inside the solar cell, and a lumped series resistance $R_{\mathrm{s}}$ represents the voltage drop of semiconductor and contacts of solar cells, respectively. And the photon-generated current $I_{\mathrm{ph}}$ is expressed by the circuit source and is related with solar irradiance as

$I_{p h}=\left(I_{s c, \text { STC }}+k_{i} \Delta T\right) \frac{G}{G_{S T C}} \frac{R_{s h}+R_{s}}{R_{s h}}$

where $I_{\mathrm{sc}, \mathrm{STC}}$ is the cell's short-circuit current in standard test conditions (STC), $k_{\mathrm{i}}=0.0017 \mathrm{~A} /{ }^{\circ} \mathrm{C}$ is cell's short current temperature coefficient, $G$ is the irradiance reaching the surface of the cell, and $\Delta \mathrm{T}=T-T_{S T C}$, where $T$ is the temperature of the PV module. In STC, the spectral conditions are AM1.5, the module temperature $\mathrm{T}_{\mathrm{STC}}$ is $25^{\circ} \mathrm{C}$, and the irradiance $\mathrm{G}_{\mathrm{STC}}$ is $1000 \mathrm{~W} / \mathrm{m}^{2}$. Since the value of $R_{\mathrm{sh}}$ is always much more larger than the value of $R_{\mathrm{s}}$, the fraction $\left(R_{s h}+R_{s}\right) / R_{s h}$ could be approximate to 1 . Thus equation (1) could be write as

$I_{p h}=\left(I_{s c, \text { STC }}+k_{i} \Delta T\right) \frac{G}{G_{S T C}}$

The current-voltage relation of a solar cell based on the one-diode circuit model can be written as

$I=I_{p h}-I_{0}\left[e^{\frac{q\left(V+I R_{s}\right)}{n k T}}-1\right]-\frac{V+I R_{s}}{R_{s h}}$

The three items for the right part of equation (3) from left to right represent the photon-generated current, diode's current and resistor's $\left(R_{\mathrm{sh}}\right)$ current, re- spectively. $I_{0}$ is the saturation current of the diode, $k=1.38 \times 10^{-23} \mathrm{~J} / \mathrm{K}$ is Boltzmann constant, $q=1.6 \times 10^{-19} \mathrm{C}$ is the electronic charge; $n$ is the ideality factor of the $\mathrm{p}-\mathrm{n}$ junction of the diode.

\subsection{Electrical configuration of a module}

Figure 2(a) shows a conventional photovoltaic module which consists of 60 solar cells connected in series And it is protected against hot spots by three bypass diodes, each of them connected in antiparallel with 20 cells. Figure 2(b) shows an improved module which is introduced in section 3.2. It is seen from Figure 2 that the area of a module is divided into three blocks. In this simulation, shading of the array is done one single block rather than one single module.

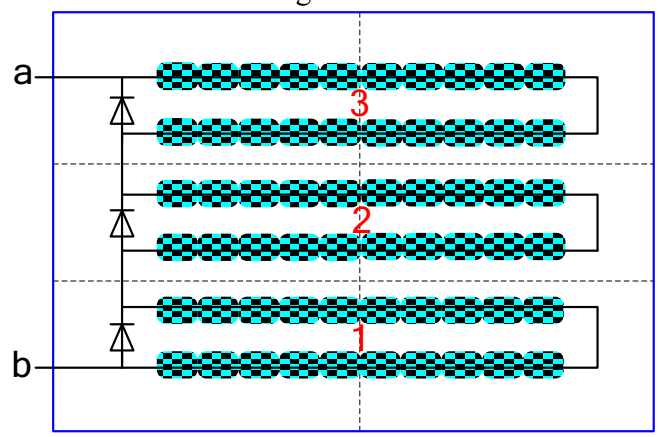

(a)

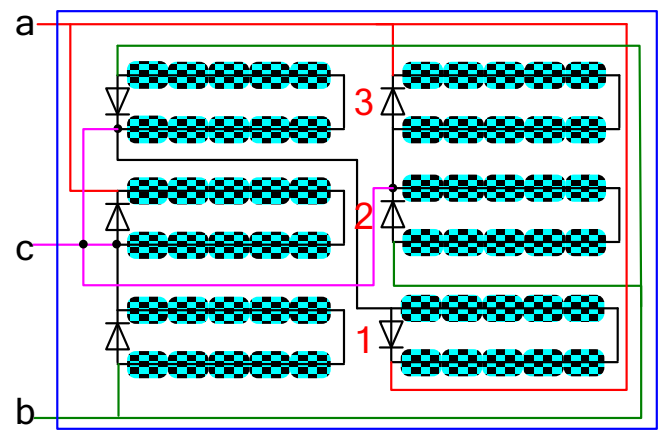

(b)

Figure2. (a) Electrical configuration of a conventional photovoltaic module. (b) Electrical configuration of an improved photovoltaic module.

\subsection{Interconnection scheme of the array}

In the simulation, a $3 \times 3 \mathrm{PV}$ array with its modules interconnected with both TCT and static schemes are employed, as shown in Figures 3(a) and (b), respectively, and the physical placement of array is shown in Figure 3(c).

In the Figure, $\mathrm{M}$ refers to the module, and the subscript denotes the physical position of the module in the array, for example $\mathrm{M}_{12}$ refers to the module in row 1 and in column 2. With TCT scheme, modules in the 
same row are connected in parallel and then the rows are connected in series, thus the electrical arrangement of array is the same as its physical placement, seen in Figures 3(a) and (c). And with static scheme, array's electrical arrangement is different from its physical placement since modules of different rows connected in parallel, as shown in Figures 3(b) and (c). Thus, when the array is partially shaded by a shadow, seen in Figure 3(c), the shaded modules in array with TCT scheme are in the same row of its electrical arrangement while those in array with static scheme are in different rows, seen in Figures 3(a) and (b). Thus, the effect of shading is distributed over the static interconnected array thereby reducing the occurrence of shading of modules in the same row and enhancing the maximum power generated from photovoltaic array $[14,15]$.

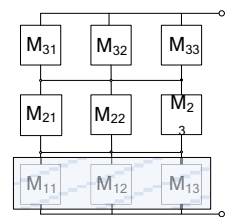

(a)

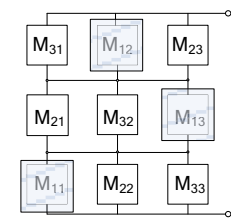

(b)

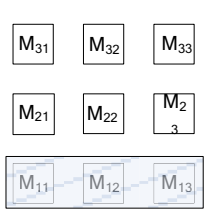

(c)
Figure 3. Sketch map of (a) array with TCT interconnection,

(b) array with the static interconnection, and (c) array's physical placement

\subsection{Implement Method}

The simulation is implemented in PSpice, which is a conventional circuit simulator and is a natural model to predict power generated from PV array since the solar cells are usually represented by a one-diode circuit model. Moreover, the interconnection schemes among modules and the electrical connection of cells in the single module could be easily and conveniently optimized in the simulation environment of PSpice. In this simulation, a sub-circuit is defined, as seen in Figure 4. In the sub-circuit, the solar cell is represented by the one-diode model and bypass diodes is connected in antiparallel with several solar cells. With the conventional module, the number of series connected solar cells of the sub-circuit is 20 , and with the improved module the number of cells of the sub-circuit is 10. In the sub-circuit, the illumination of each solar cell is the same and the photo-current source is controlled by the voltage source $V_{\mathrm{G}}$ as

$$
I_{p h}=\left(I_{s c, \text { STC }}+k_{i} \Delta T\right) \frac{V_{G}}{1000}
$$

Where 1000 refers to $1000 \mathrm{~W} / \mathrm{m}^{2}$ which is the irradiance in STC, and the $V_{\mathrm{G}}$ represents the illumination of solar cells. So if the voltage of $V_{\mathrm{G}}$ is $200 \mathrm{~V}$, the solar cells shaded and the illumination is $200 \mathrm{~W} / \mathrm{m}^{2}$. After the sub-circuit are "built", the circuit model of array could be constructed. And as described in section 2.2, three sub-circuits are needed to construct a conventional module while six sub-circuit are used to construct an improved module.

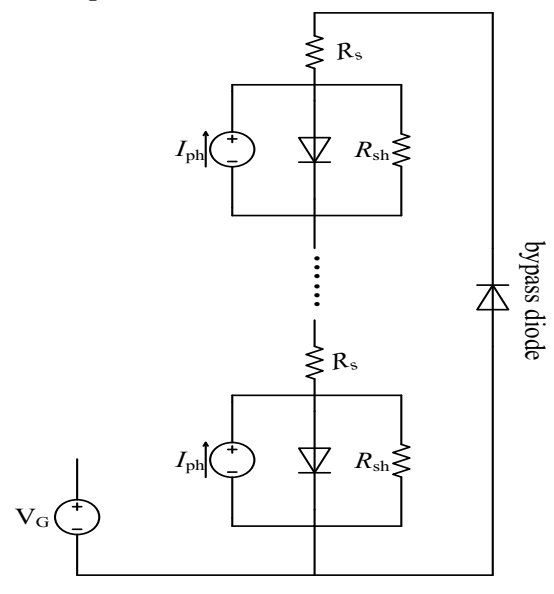

Figure 4. Sketch map of the sub-circuit.

\section{SIMULATION RESULTS AND ANALYSIS}

In the simulation, all cell parameters were obtained by fitting the one-diode model to the current-voltage characteristics of a real solar cell. The cell parameters are as follows: $J_{\mathrm{SC}}=39.9 \mathrm{~mA} / \mathrm{cm}^{2}, J_{0}=10^{-11} \mathrm{~A} / \mathrm{cm}^{2}$, $\mathrm{n}=1.1, R_{\mathrm{s}}=0.5 \Omega \cdot \mathrm{cm}^{2}, R_{\mathrm{sh}}=2.4 \times 10^{5} \Omega \cdot \mathrm{cm}^{2}, T=298 \mathrm{~K}$, and the cell area is $126.6 \mathrm{~cm}^{2}$. And the illuminations for unshaded and shaded cells are $200 \mathrm{~W} / \mathrm{m}^{2}$ and $1000 \mathrm{~W} / \mathrm{m}^{2}$, respectively.

\subsection{MPP of photovoltaic array under partial shading} and with different interconnection schemes

The coordinate graph of a $3 \times 3 \mathrm{PV}$ array is shown in Figure5. As seen in the Figure, length and width of the $3 \times 3$ array is $9 a$ and $3 b$, respectively. And the shape of shadow is described by its length and width, e.g. the length and width of shadow in Figure 5 is $4 \mathrm{a}$ and $2 \mathrm{~b}$, respectively. It is easily seen from the Figure that illumination on some modules are partially shaded (e.g. module in row2, column1 and module in row2, column2) with the shadow, whose shape is common.

Figure 6 shows the plots of maximum output power generated from the array against shadow's length for both TCT and static interconnection schemes, and the width of shadow for (a), (b), (c), is $1 b, 2 b$, and $3 b$ respectively. Symbol " $\square$ " represents the maximum power generated from array with TCT interconnection while "O" represents the maximum power generated from array with static interconnection.

In Figure 6(a), the maximum output power obtained from array with static scheme is the same as those obtained from array with TCT scheme, and this is as predicted since in that case the distribution of effect of shading is not actually occurred. In Figure 6(b), the maximum output power obtained from array with static scheme is higher than those obtained from array 


\section{MATEC Web of Conferences}

with TCT scheme, except the case that shadow's length is 1a. In Figure 6(c), the maximum output power obtained from array with static scheme when shadow's length is 1a and $4 \mathrm{a}$ are $895.7 \mathrm{~W}$ and $578.4 \mathrm{~W}$, which are lower than those obtained from array with TCT scheme $(1059.8 \mathrm{~W}$ and $596.9 \mathrm{~W}$ for shadow's length are $1 \mathrm{a}$ and $4 \mathrm{a}$, respectively). As reported in [14, $15]$, the power produced with static scheme is equal to the TCT scheme under worst conditions. However, this simulation shows that the power generated with static scheme maybe lower than those generated with TCT scheme if the illumination of the module on the edge of shadow is non-uniform, for example, the cases of shadow's length is $1 \mathrm{a}$ and $4 \mathrm{a}$.

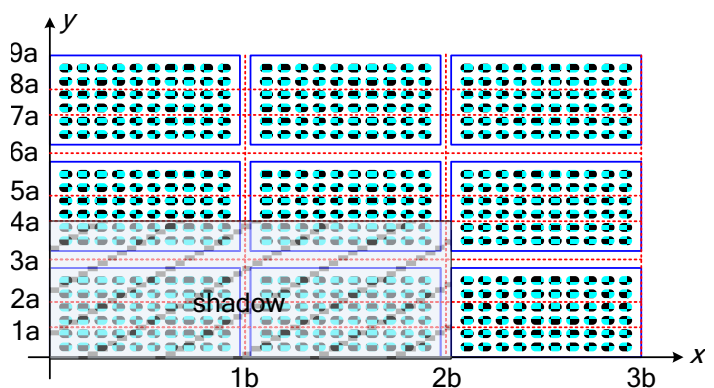

Figure 5. Coordinate graph of a $3 \times 3 \mathrm{PV}$ array assuming that there is no space between two adjacent cells and modules.
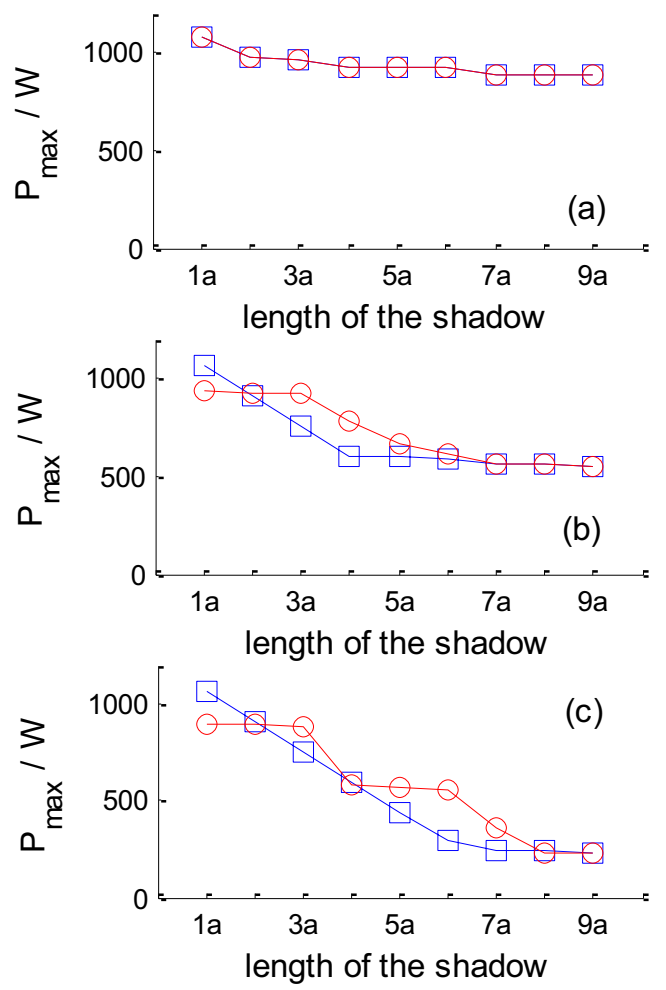

Figure 6. Plots of maximum power generated from the array against shadow's length for both TCT (“ $\square$ ”) and the static ("O") interconnection schemes, and the width of shadow for (a), (b), (c), is $1 b, 2 b$, and $3 b$, respectively.

\subsection{MPP of photovoltaic array under partial shading and with different modules}

In order to increase the maximum power generated from array with static scheme when the illumination of modules on the edge of shadow is nonuniform, an improved electrical configuration of a module is presented in Figure 2(b). The improved module consists of two strings in a bunch with each string consists of a bypass diode connected in antiparallel with 10 cells, and then three bunches are connected ties across each junction. Figures 7(a) and (b) show the electrical arrangement of a $3 * 3$ array equipped with the conventional module and improved module, respectively. In the Figure, the square refers to the module and the rounded rectangle refers to the string.

(a)

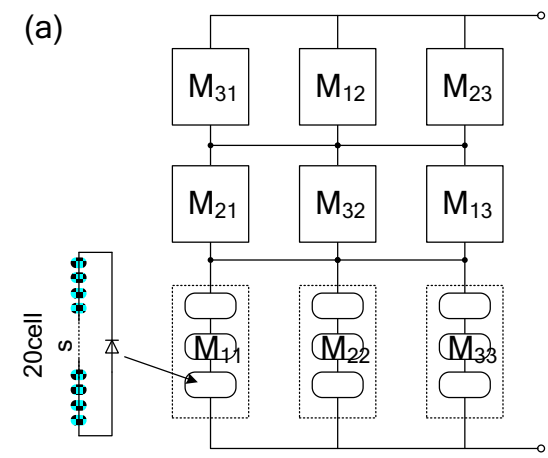

(b)

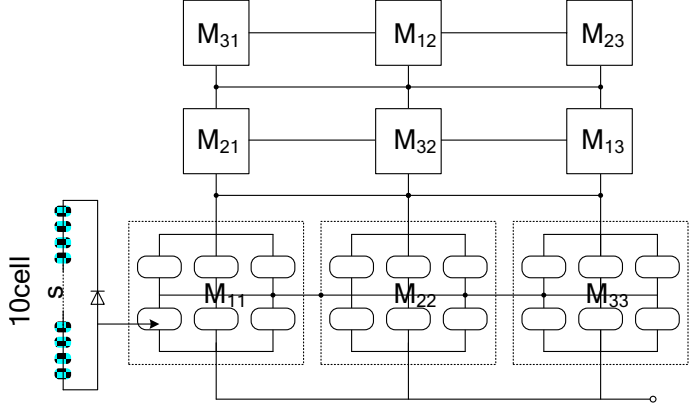

Figure 7. Electrical arrangement of the $3 \times 3$ array which is equipped with (a) conventional module and (b) improved module

As shown in Figure 7, array with the conventional module is totally cross tied in the module level while array with the improved module is totally cross tied in the string level. So the string in Figure 7(b) is played the role of module in Figure 7(a) and the string is always completely shaded or unshaded due to the fact 
that when one cell of a string is completely shaded, the whole string will be inactive (situations that the cell is partially shaded are not considered here). As a consequence, the circumstance discussed in section 3.1 of the nonuniform illumination of modules on the edge of shadow is avoided. Figure 8 gives the plot of maximum power against the shadow's length for array with static scheme and equipping conventional modules (“○”) and improved modules (“※”), respectively.
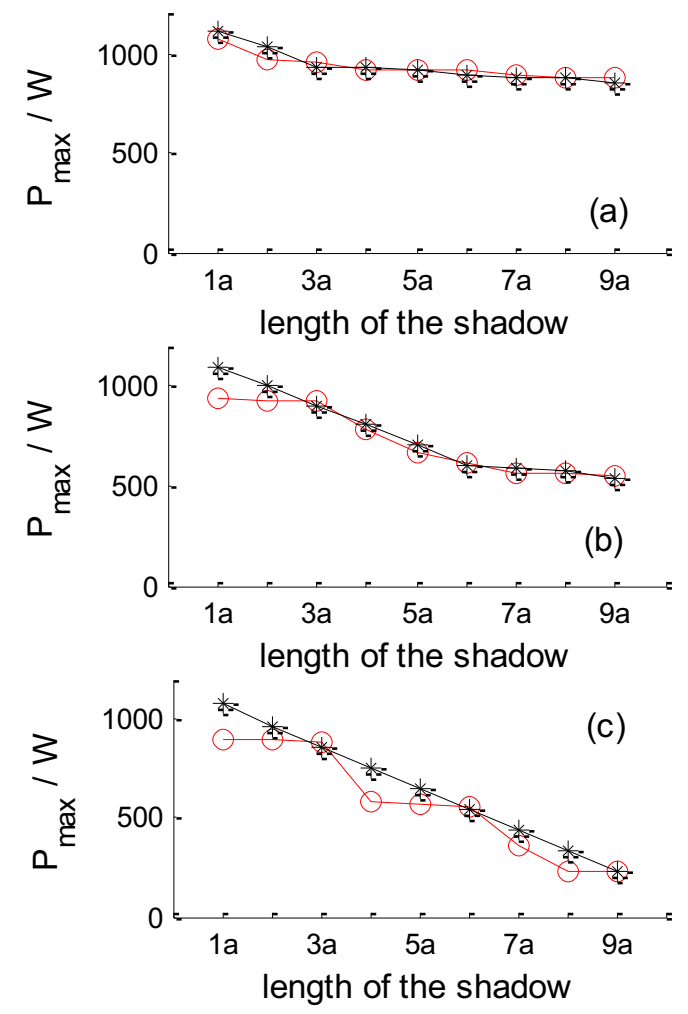

Figure 8. Plots of maximum power against the shadow's length for array with static scheme, equipping conventional modules (“०”) and improved modules (“※”), respectively. And the width of shadow for (a), (b), (c) is $1 b, 2 b$, and $3 b$, respectively.

It is seen form Figure 8 that when illumination of modules on the edge of shadow is non-uniform (in cases of shadow's length is $1 \mathrm{a}, 2 \mathrm{a}, 4 \mathrm{a}, 5 \mathrm{a}, 7 \mathrm{a}$, and 8a), the maximum power generated from array with static scheme is enhanced by equipping the improved modules, especially in the case of wide-shape shadow shown in Figure 8(c). However, it should be noticed that the voltage at which the output power generated form array achieves at its maximum value is evidently decreased by introducing improved modules since more parallel connected strings are employed. Figure 8 shows the power-voltage curve obtained from array equipping conventional modules and improved mod- ules, when shadow's length and width are $1 \mathrm{a}$ and $3 \mathrm{~b}$, respectively. As seen in Figure 9, the voltage of the maximum power point is $27.4 \mathrm{~V}$, where array equipping improved modules has a power of $1070.5 \mathrm{~W}$. Meanwhile, the maximum power of array equipping conventional modules is around $85.3 \mathrm{~V}$ with a power of $895.7 \mathrm{~W}$.

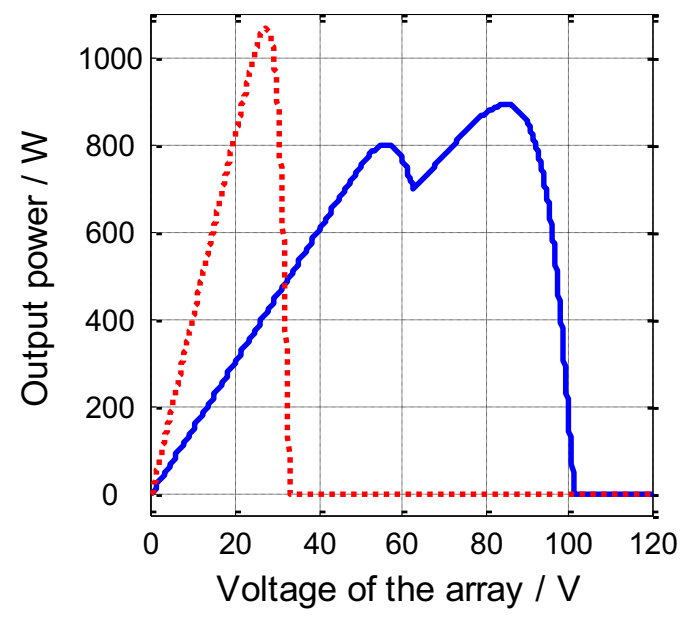

Figure 9. Plot of power generated from the array against array's voltage.

In the Figure 9, power generated from array equipping conventional module is presented by the solid line while that from array equipping improved modules is presented by the dotted line.

\section{CONCLUSIONS}

It is confirmed that the maximum power generated from photovoltaic array is decreased under partial shading conditions through different mechanisms and could be enhanced by optimizing the interconnection scheme of array. The static scheme is improved from TCT scheme and is designed to distribute the effect of shading over array. In previous report, the power produced with static scheme is equal to the TCT scheme even under worst conditions. However, in those reports the illumination of a single module is assumed to be uniform, while in urban environments the illumination of modules on the edge of shadow is likely to be non-uniform. In this paper, the module is divided into three blocks according to the number of bypass diodes, and shading of the array is done one single block rather than one single module. And the simulation results show that the power generated with static scheme maybe lower than those generated with TCT scheme if the illumination of the module on the edge of shadow is non-uniform. Then the electrical configuration of a module is optimized to increase the array's maximum power when the illumination of modules on the edge of shadow is non-uniform. At last, simulation of this 


\section{MATEC Web of Conferences}

paper is implemented in PSpice, with which the design and optimization of interconnection scheme of array as well as electrical connection of module could be done conveniently.

\section{ACKNOWLEDGEMENT}

This work is sponsored by the Basic Research Program of Shenzhen (NO. JCYJ20130329102120434)

\section{REFERENCES}

[1] S. Silvestre. \& A. Chouder. 2007. Effects of shading on photovoltaic module performance. Progress in Photovoltaics: Research and Applications, 16(2): 141-149.

[2] A. Woyte, J. Nijs, \& R. Belmans. 2003. Partial shadowing of photovoltaic arrays with different system configurations: Literature review and field test results. Solar Energy, 74(3): 217-233.

[3] E. Koutroulis. \& F. Blaabjerg. 2012. A new technique for tracking the global maximum power point of PV arrays operating under partial-shading conditions. IEEE Journal of Photovoltaics, 2(2): 184-190.

[4] U. Zimmermann. \& M. Edoff. 2012. A maximum power point tracker for long-term logging of PV module performance. IEEE Journal of Photovoltaics, 2(1): 47-55.

[5] M. Uno. \& A. Kukita. 2015. Single-Switch Voltage Equalizer Using Multistacked Buck-Boost Converters for partially shaded photovoltaic modules. IEEE Transactions on Power Electronics, 30(6): 3091-3105.

[6] C. E. Chamberlin, P. Lehman. \& J. Zoelick, et al. 1995. Effects of mismatch losses in photovoltaic arrays. Solar Energy, 54(3): 165-171.

[7] N. D. Kaushika. \& A. K. Rai. 2007. An investigation of mismatch losses in solar photovoltaic cell networks. Energy, 32(5): 755-759.

[8] J. Moein, U. Sener, \& J. Kian. 2014. A comparative study on different photovoltaic array topologies under partial shading conditions. T\&D Conference and Exposition, 2014 IEEE PES. Publication Year: Pages: 1-5.

[9] A. Maki. \& S. Valkealahti. 2012. Power losses in long string and parallel-connected short strings of series-connected silicon-based photovoltaic modules due to partial shading conditions. IEEE Transactions of Energy Conversion, 27(1): 173-183.

[10]S. Khatoon, Ibraheem, \& M. F. Jalil. 2014. Analysis of solar photovoltaic array under partial shading conditions for different array configurations. Computational Intelligence on Power, Energy and Controls with their impact on Humanity (CIPECH), 2014 Innovative Applications of. Publication Year: 2014. Pages: 452-456.

[11]L.F.L. Villa, D. Picault. \& S. Bacha, et al. 2012. Maximizing the power output of partially shaded photovoltaic plants through optimization of the interconnections among its modules. IEEE Journal of Photovoltaics, 2(2):154-163.

[12]M. Z. Shams El-Dein, Mehrdad Kazerani, \& M.M.A. Salama. 2013. An optimal photovoltaic array reconfigu- ration to reduce partial shading losses. IEEE Transactions on Sustainable Energy, 4(1): 145-153.

[13]D. Nguyen \& B. Lehman. 2008. An adaptive solar photovoltaic array using model-based reconfiguration algorithm. IEEE Transactions on Industrial Electronics. 55(7): 2644-2654.

[14]B. Indu Rani, G. Saravana Ilango, \& Chilakapati Nagamani. 2013. Enhanced Power Generation From PV Array Under Partial Shading Conditions by Shade Dispersion Using Su Do Ku Configuration. IEEE Transaction on sustainable energy. 4(3):594-601.

[15]P. Srinivasa Rao, G. Saravana Ilango, \& Chilakapati Nagamani. 2014. Maximum power from PV arrays using a fixed configuration under different shading conditions. IEEE Journal of Photovoltaics, 4(2): 679-686.

[16]S. Guo, T. Michael Walsh. \& Armin G. Aberle, et al. 2012. Analysing partial shading of PV modules by circuit modeling. Photovoltaic Specialists Conference (PVSC), 2012 38th IEEE. Publication Year: 2012. pp: 002957-002960. 